\title{
Two-Way Teleportation
}

\author{
A. Grudka* and R.W. ChHajlany \\ Faculty of Physics, Adam Mickiewicz University \\ Umultowska 85, 61-614 Poznań, Poland
}

(Received August 18, 2003)

\begin{abstract}
A protocol for teleporting two qudits simultaneously in opposite directions using a single pair of maximally entangled qudits is presented. This procedure works provided that the product of dimensions $d_{1}$ and $d_{2}$ of the two qudits to be teleported does not exceed the dimension $d$ of the individual qudits in the maximally entangled pair.
\end{abstract}

PACS numbers: 03.67.-a

\section{Introduction}

Quantum teleportation, the process of transferring complete information about a quantum state from a sender to a receiver, is one of the fundamental features of quantum information. In the original setting [1], Alice and Bob utilize a shared maximally entangled pair of qubits to teleport a qubit in some unknown state from Alice to Bob. This is implemented by local operations by Alice and Bob supplemented by classical communication between them. This scheme is easily generalized to the case of qudits ( $d$-dimensional quantum systems) $[1,2]$. In order to teleport a $d$-dimensional qudit, Alice and Bob must share a pair of at least $d$-dimensional qudits in the maximally entangled state. Quantum teleportation between multiple parties using qubits $[3,4]$ and qudits [5,6] has also been widely studied. In all teleportation protocols, the pair of maximally entangled qudits plays the role of a uni-directional quantum channel.

In this article we analyze two-way quantum teleportation, i.e. simultaneous teleportation of two qudits in two opposite directions, as introduced in [7]. Let us suppose that Alice possesses a $d_{1}$-dimensional qudit in the state $|\alpha\rangle_{1}=$ $\sum_{i=0}^{d_{1}-1} \alpha_{i}|i\rangle_{1}$, while Bob has a $d_{2}$-dimensional qudit in the state $|\beta\rangle_{2}=\sum_{i=0}^{d_{2}-1} \beta_{i}|i\rangle_{2}$.

* corresponding author; e-mail: agie@amu.edu.pl 
The qudits 1 and 2 will henceforth be called teleportee qudits. Additionally Alice and Bob share a maximally entangled pair of $d$-dimensional qudits, which serves the purpose of a quantum channel, in the state

$$
|\Psi\rangle=\frac{1}{\sqrt{d}} \sum_{i=0}^{d-1}|i\rangle_{c 1}|i\rangle_{c 2}
$$

such that $d_{1} d_{2} \leq d$. In accordance with the notation, the qudits in the state $|\Psi\rangle$ will be referred to as channel qudits with the first qudit belonging to Alice and the second to Bob. By applying the standard teleportation protocol of Bennett et al., Alice and Bob can manage to teleport only one of the two states $|\alpha\rangle$ or $|\beta\rangle$ using the channel described by Eq. (1). We construct an explicit scheme enabling the teleportation of $|\alpha\rangle_{1}$ from Alice to Bob and $|\beta\rangle_{2}$ from Bob to Alice using the shared maximally entangled pair in the state $|\Psi\rangle$.

\section{The protocol}

Our teleportation scheme consists of three main stages: (1) tailoring of the quantum channel, (2) encoding of information about the states to be teleported into the channel qudits and (3) decoding information in the channel into qudits in product form. We divide our considerations into two cases, viz. $d_{1} d_{2}=d$ and $d_{1} d_{2}<d$.

We first consider the former case, where the quantum channel is characterized by the dimension $d=d_{1} d_{2}$. In this case, the channel is already in the desired state. The first step of the encoding stage consists of Alice projecting her two qudits into one of $d_{1}$ orthogonal $d$-dimensional subspaces of the original $d_{1} d$-dimensional space, by measuring the projectors

$$
\begin{aligned}
P_{k_{1}} & =\sum_{j_{1}=0}^{d_{1}-1} \sum_{j_{2}=0}^{d_{2}-1}\left|j_{1}\right\rangle_{1}\left|\left(j_{1} \oplus_{1} k_{1}\right)+j_{2} d_{1}\right\rangle_{c 1 c 1}\left\langle\left(j_{1} \oplus_{1} k_{1}\right)+j_{2} d_{1}\right|{ }_{1}\left\langle j_{1}\right| \\
& k_{1}=0, \ldots, d_{1}-1
\end{aligned}
$$

where $\oplus_{1}$ denotes addition modulo $d_{1}$. Bob performs an analogous measurement on his qudits with $d_{2}$ outcomes, $k_{2}=0, \ldots, d_{2}-1$, described by

$$
P_{k_{2}}=\sum_{j_{1}=0}^{d_{1}-1} \sum_{j_{2}=0}^{d_{2}-1}\left|j_{2}\right\rangle_{2}\left|j_{1}+\left(j_{2} \oplus_{2} k_{2}\right) d_{1}\right\rangle_{c 2 c 2}\left\langle j_{1}+\left.\left(j_{2} \oplus_{2} k_{2}\right) d_{1}\right|_{2}\left\langle j_{2}\right|,\right.
$$

with $\oplus_{2}$ denoting addition modulo $d_{2}$. If Alice and Bob obtain the results $k_{1}$ and $k_{2}$ respectively, then the joint state of all four qudits considered is

$$
\begin{aligned}
\left|\psi_{1}\right\rangle & =\sum_{j_{1}=0}^{d_{1}-1} \sum_{j_{2}=0}^{d_{2}-1} \alpha_{j_{1}} \beta_{j_{2}}\left|j_{1}\right\rangle_{1} \mid\left(j_{1} \oplus_{1} k_{1}\right) \\
& \left.+\left(j_{2} \oplus_{2} k_{2}\right) d_{1}\right\rangle_{c 1}\left|j_{2}\right\rangle_{2}\left|\left(j_{1} \oplus_{1} k_{1}\right)+\left(j_{2} \oplus_{2} k_{2}\right) d_{1}\right\rangle_{c 2}
\end{aligned}
$$


Next, Alice and Bob inform each other about the results of their local measurements. Using these results, they perform the following operation on their channel qudits:

$$
\left|\left(j_{1} \oplus_{1} k_{1}\right)+\left(j_{2} \oplus_{2} k_{2}\right) d_{1}\right\rangle_{c r} \rightarrow\left|j_{1}+j_{2} d_{1}\right\rangle_{c r},
$$

where $r=1,2$ refer to Alice's and Bob's qudits respectively. The state $\left|\psi_{1}\right\rangle$ is transformed to

$$
\left.\left|\psi_{2}\right\rangle=\sum_{j_{1}=0}^{d_{1}-1} \sum_{j_{2}=0}^{d_{2}-1} \alpha_{j_{1}} \beta_{j_{2}}\left|j_{1}\right\rangle_{1}\left|\left(j_{1}+j_{2} d_{1}\right\rangle_{c 1}\right| j_{2}\right\rangle_{2}\left|j_{1}+j_{2} d_{1}\right\rangle_{c 2} .
$$

Now Alice and Bob perform the following unitary operation on their respective pairs of qudits:

$$
\left|j_{r}\right\rangle_{r}\left|j_{1}+j_{2} d_{1}\right\rangle_{e r} \rightarrow|0\rangle_{r}\left|j_{1}+j_{2} d_{1}\right\rangle_{c r}
$$

that leaves the first qudit of each pair in a standard state $|0\rangle_{r}(r=1,2)$ and the channel qudit unchanged. In this way, entire information about the teleportee states is encoded into the quantum channel, which is in the state

$$
\left|\psi_{3}\right\rangle=\sum_{j_{1}=0}^{d_{1}-1} \sum_{j_{2}=0}^{d_{2}-1} \alpha_{j_{1}} \beta_{j_{2}}\left|j_{1}+j_{2} d_{1}\right\rangle_{c 1}\left|j_{1}+j_{2} d_{1}\right\rangle_{c 2},
$$

while the other two qudits can be discarded. The two parties now share the information about both states $|\alpha\rangle$ and $|\beta\rangle$.

To complete the teleportation procedure, Alice and Bob have to decode the information present in $\left|\psi_{3}\right\rangle$. To do this, Alice performs a $d_{1}$-dimensional Fourier transform described below

$$
\left|j_{1}+j_{2} d_{1}\right\rangle_{c 1} \rightarrow \frac{1}{\sqrt{d_{1}}} \sum_{m_{1}=0}^{d_{1}-1} \omega_{d_{1}}^{m_{1} j_{1}}\left|m_{1}+j_{2} d_{1}\right\rangle_{c 1}
$$

where $\omega_{d_{1}}=\exp \left[i 2 \pi / d_{1}\right]$ is complex root of unity. Bob performs a similar Fourier transform on his channel qudit

$$
\left|j_{1}+j_{2} d_{1}\right\rangle_{c 2} \rightarrow \frac{1}{\sqrt{d_{2}}} \sum_{m_{2}=0}^{d_{2}-1} \omega_{d_{2}}^{m_{2} j_{2}}\left|j_{1}+m_{2} d_{1}\right\rangle_{c 2} .
$$

The state of the two channel qudits is transformed in this way into

$$
\begin{aligned}
\left|\psi_{4}\right\rangle & =\frac{1}{\sqrt{d}} \sum_{m_{1}=0}^{d_{1}-1} \sum_{j_{1}=0}^{d_{1}-1} \sum_{m_{2}=0}^{d_{2}-1} \sum_{j_{2}=0}^{d_{2}-1} \alpha_{j_{1}} \beta_{j_{2}} \omega_{d_{1}}^{m_{1} j_{1}} \omega_{d_{2}}^{m_{2} j_{2}} \\
& \times\left|m_{1}+j_{2} d_{1}\right\rangle_{c 1}\left|j_{1}+m_{2} d_{1}\right\rangle_{c 2} .
\end{aligned}
$$

Alice and Bob disentangle their qudits by performing the projections

$$
Q_{m_{1}}=\sum_{j_{2}=0}^{d_{2}-1}\left|m_{1}+j_{2} d_{1}\right\rangle\left\langle m_{1}+j_{2} d_{1}\right|, m_{1}=0, \ldots, d_{1}
$$


and

$$
Q_{m_{2}}=\sum_{j_{1}=0}^{d_{1}-1}\left|j_{1}+m_{2} d_{1}\right\rangle\left\langle j_{1}+m_{2} d_{1}\right|, m_{2}=0, \ldots, d_{2}
$$

on their respective qudits. Any pair of results $m_{1}, m_{2}$ of these local measurements occurs with equal probability $d^{-1}$ yielding the product state

$$
\left|\psi_{5}\right\rangle=\sum_{j_{1}=0}^{d_{1}-1} \sum_{j_{2}=0}^{d_{2}-1} \alpha_{j_{1}} \beta_{j_{2}} \omega_{d_{1}}^{m_{1} j_{1}} \omega_{d_{2}}^{m_{2} j_{2}}\left|m_{1}+j_{2} d_{1}\right\rangle_{c 1}\left|j_{1}+m_{2} d_{1}\right\rangle_{c 2} .
$$

Alice and Bob communicate the results of the measurements $Q_{m_{1}}$ and $Q_{m_{2}}$ (Eqs. (12) and (13)) to one another and perform the unitary operations $U_{1}$ and $U_{2}$ :

$$
\begin{aligned}
& U_{1}\left|m_{1}+j_{2} d_{1}\right\rangle_{c 1}=\omega_{d_{2}}^{-m_{2} j_{2}}\left|j_{2} d_{1}\right\rangle_{c 1} \\
& U_{2}\left|j_{1}+m_{2} d_{1}\right\rangle_{c 2}=\omega_{d_{1}}^{-m_{1} j_{1}}\left|j_{1}\right\rangle_{c 2}
\end{aligned}
$$

on their qudits. The channel qudits end up in the state

$$
\left|\psi_{6}\right\rangle=\sum_{j_{2}=0}^{d_{2}-1} \beta_{j_{2}}\left|j_{2} d_{1}\right\rangle_{c 1} \sum_{j_{1}=0}^{d_{1}-1} \alpha_{j_{1}}\left|j_{1}\right\rangle_{c 2}=\left(V_{1}^{\dagger}|\beta\rangle_{c 1}\right)|\alpha\rangle_{c 2} .
$$

Bob's qudit is now in the desired state $|\alpha\rangle$ while Alice's qudit completely encodes the state $|\beta\rangle$. Alice only has to perform a rotation of basis $V_{1}\left|j_{2} d_{1}\right\rangle_{c 1}=$ $\left|j_{2}\right\rangle_{c 1}$ to exactly retrieve the original state. In this manner, teleportation in two opposite directions is achieved.

Let us notice that teleportation of both states in this process can essentially be conducted simultaneously. This process is successful with unit efficiency since it is implemented using only unitary operations and complete measurements.

Now, let us consider the case when $d_{1} d_{2}=d^{\prime}<d$. In this case, the protocol presented above cannot be directly applied. The reason behind this is that the projections applied do not span the entire local spaces of the given qudits, which in turn may lead to a loss of information due to the possible projection onto some complementary space. The conversion of the state of the given channel (Eq. (1)) into the required form can be conducted beforehand by Alice and Bob in a "tailoring" stage, as discussed below.

Alice (or Bob) can prepare an ancillary $d^{\prime}$-dimensional qudit in the state

$$
|\phi\rangle=\frac{1}{\sqrt{d^{\prime}}} \sum_{i=0}^{d^{\prime}-1}|i\rangle_{0}
$$

e.g. by applying the $d^{\prime}$-dimensional Fourier transform to the state $|0\rangle_{0}$. The joint state of the ancilla and channel qudits is $|\phi\rangle|\Psi\rangle$. Alice performs a measurement having $d$ outcomes defined by the projection operators

$$
R_{k}=\sum_{n=0}^{d^{\prime}-1}|n\rangle_{0}|n+k\rangle_{c 1 c 1}\left\langle n+\left.k\right|_{0}\langle n|\right.
$$


where $k=0,1, \ldots d-1$ and all sums are taken modulo $d$. The state $|\phi\rangle|\Psi\rangle$ is projected with equal probability $d^{-1}$ into one of the states

$$
\left|\varphi_{k}\right\rangle=\frac{1}{\sqrt{d^{\prime}}} \sum_{n=0}^{d^{\prime}-1}|n\rangle_{0}|n+k\rangle_{c 1}|n+k\rangle_{c 2} .
$$

Alice unitarily separates the ancilla from her channel qudits by the operation

$$
|n\rangle_{0}|n+k\rangle_{e 1} \rightarrow|0\rangle_{0}|n+k\rangle_{e 1}
$$

Alice sends the result $k$ to Bob, and both parties perform the subtraction operation $|i\rangle_{c r} \rightarrow|i-k\rangle_{c r}$ leaving the channel qudits in the maximally entangled states

$$
\left|\Psi^{\prime}\right\rangle=\frac{1}{\sqrt{d^{\prime}}} \sum_{i=0}^{d^{\prime}-1}|i\rangle_{c 1}|i\rangle_{c 2} .
$$

This pair of qudits can now be used as a channel for teleporting two qudits in opposite directions, by performing steps (2) and (3) as described earlier.

\section{Concluding remarks}

A general protocol utilizing a maximally entangled state as a bi-directional channel for teleportation was presented. The only condition for successful teleportation is that the information to be exchanged occupies a space of at most as high a dimension as that occupied by each channel qudit.

One of us (A.G.) would like to thank the State Committee for Scientific Research for financial support under grant No. 0 T00A 00323.

\section{References}

[1] C.H. Bennett, G. Brassard, C. Crepeau, R. Jozsa, A. Peres, W.K. Wootters, Phys. Rev. Lett. 70, 1895 (1993).

[2] S. Stenholm, P.J. Bardroff, Phys. Rev. A 58, 4373 (1998).

[3] A. Karlsson, M. Bourennane, Phys. Rev. A 58, 4394 (1998).

[4] M. Hillery, V. Bužek, Berthiaume, Phys. Rev. A 59, 1829 (1999).

[5] M. Murao, M.B. Plenio, V. Vedral, Phys. Rev. A 61, 032311 (2000).

[6] I. Ghiu, Phys. Rev. A 7, 012323 (2003).

[7] A. Grudka, Los Alamos archive quant-ph/0303112. 